\title{
Ingenol Mebutate: An Emerging Therapy in the Treatment of Actinic Keratoses
}

\author{
Stephanie Jacks • Kasie Kudrewicz • David R. Carr
}

Published online: 7 April 2013

(C) Springer Science+Business Media New York 2013

\begin{abstract}
Ingenol mebutate gel, derived from the sap of the Euphorbia peplus plant, recently has been investigated as a potential topical treatment for actinic keratoses. The medication is proposed to have a dual mechanism of action: rapid lesion necrosis followed by lesion-specific, neutrophilmediated, antibody-dependent cellular cytotoxicity. A literature review of the data from phase II and III clinical trials reveals that ingenol mebutate gel is an efficacious and safe therapy for actinic keratoses when used on areas of skin up to $25 \mathrm{~cm}^{2}$. Further research is needed to compare directly ingenol mebutate gel to other treatments available for actinic keratoses, as well as to establish safety and efficacy for application on larger areas of skin.
\end{abstract}

Keywords Ingenol mebutate $\cdot$ Actinic keratosis ·

Euphorbia peplus

\section{Introduction}

Actinic keratoses are premalignant lesions commonly encountered in dermatology, with risk factors that include fair skin types, age, and a history of chronic sun exposure [1]. Cryotherapy is the most widely utilized treatment, but it is associated with the risk of scarring. Topical therapies, such

\author{
S. Jacks $\cdot$ D. R. Carr $(\bowtie)$ \\ Division of Dermatology, Ohio State University, \\ Martha Morehouse Tower, 4th Floor, \\ Columbus, OH 43220, USA \\ e-mail: david.carr@osumc.edu \\ S. Jacks \\ e-mail: Stephanie.jacks@osumc.edu \\ K. Kudrewicz \\ Division of Dermatology, \\ Ohio University/O'Bleness Memorial Hospital, 55 Hospital Dr, \\ Athens, OH 45701, USA \\ e-mail: Kasie.Kudrewicz@gmail.com
}

as 5-fluorouracil or imiquimod, are disadvantageous for other reasons, including the longer duration of treatment and the risk of localized skin reactions with prolonged application, both of which may negatively impact patient adherence to treatment $[2,3 \cdot \bullet]$.

Recently, the medical community has focused its attention on a new treatment for actinic keratoses called ingenol mebutate. This medication is derived from the sap of the Euphorbia peplus plant, also known as petty spurge, radium weed, or milkweed. The sap is a white, sticky irritant that has long been used in traditional medicine for treatment of warts, corns, and nonmelanoma skin cancers [4]. An Australian survey from 1986 regarding the use of home remedies for skin cancers and actinic keratoses described support among respondents regarding the effectiveness of the sap of Euphorbia peplus [5]. Given the long history of use in traditional medicine, as well as the absence of documented adverse effects, recent clinical trials have been designed to further investigate the effectiveness and safety profile of ingenol mebutate.

In January 2012, ingenol mebutate gel attained FDA approval for the treatment of actinic keratoses. For areas on the face and scalp, ingenol mebutate $0.015 \%$ gel is approved for application once daily for 3 consecutive days for an area of skin up to $25 \mathrm{~cm}^{2}$. For areas on the trunk and extremities, ingenol mebutate $0.05 \%$ gel is approved for application once daily for 2 consecutive days, also for an area up to $25 \mathrm{~cm}^{2}$ [6]. The medication is a clear colorless gel that is dispensed in cartons containing either three singleuse, $0.25 \mathrm{~g}$ tubes of $0.015 \%$ ingenol mebutate gel or two single-use, 0.25 -g tubes of $0.05 \%$ gel.

\section{Mechanism of Action}

Whereas the precise mechanism of ingenol mebutate is unknown, this diterpene ester (Fig. 1) is proposed to have 


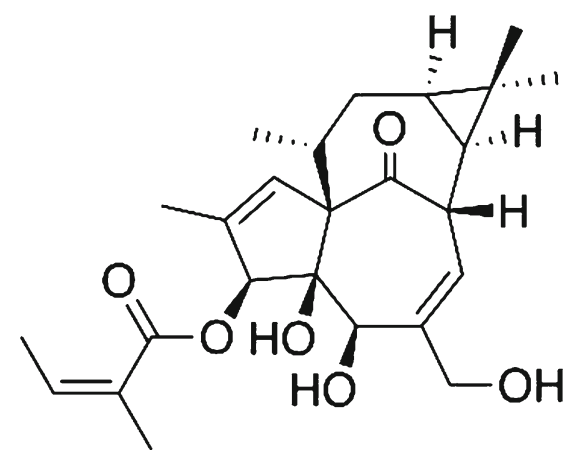

Fig. 1 Structure of ingenol mebutate (ingenol 3-angelate), an ingenol ester

a dual mechanism of action: rapid lesion necrosis followed by lesion-specific neutrophil-mediated, antibody-dependent cellular cytotoxicity (ADCC) [7••]. It is worth noting that there have been no studies of the mechanism of action of ingenol mebutate in humans, and there are no animal models for actinic keratoses, so the above hypothesis is derived from other tumor models in animals, including the B16 mouse melanoma cell line and the KL2 mouse squamous cell carcinoma cell line [8, 9].

The first aspect of the proposed mechanism of action, rapid lesion necrosis, is based on observations of changes in both the mitochondrial membranes and plasma membranes of tumor cells soon after exposure to ingenol mebutate [9]. It is hypothesized that ingenol mebutate is taken up by cells via endocytotic vesicles, which in turn release $\mathrm{Ca}^{+2}$ into the cytoplasm $[10,11]$. With the rise in intracellular $\mathrm{Ca}^{+2}$, mitochondrial membrane potential is disrupted, leading to loss of adenosine triphosphate production [7••]. Electron microscopy studies have documented swelling of the mitochondria, disruption of other organelles, and loss of plasma membrane integrity within hours of exposure to ingenol mebutate $[7 \bullet \bullet, 9]$. All of these findings lend support to the idea of necrosis, rather than apoptosis, as the mechanism of cell death. The necrosis is thought to occur quite rapidly, within 1 hour of application of ingenol mebutate [7••,9].

Neutrophil-mediated ADCC is the second aspect of the proposed mechanism. As the targeted cells undergo necrosis, the release of cytokines attracts an inflammatory infiltrate that is composed predominately of neutrophils [8]. The abundance of neutrophils at the site of treatment approximately 24 hours after application coincides with clinical evidence of inflammation, which slowly resolves over 510 days $[7 \cdot 0]$. As major players in the innate immune response, the main role of neutrophils is phagocytosis. However, neutrophils also are capable of recognizing cells coated with antibody via their $\mathrm{Fc}$ receptors, which can trigger killing of target cells through release of reactive oxygen species, a process known as ADCC. Ingenol mebutate, through unclear mechanisms, also increases production of antibodies by B cells, including tumor-specific antibodies, which likely enhances the process of neutrophilmediated ADCC $[7 \cdot \bullet, 8]$. Interestingly, in neutrophildepleted mice treated with ingenol mebutate, tumor ablation initially occurred, likely due to lesion-specific necrosis, but $83 \%$ of the mice had tumor regrowth in a period of approximately 3 months [8]. Similar relapses were noted in B celldeficient mice with severe combined immunodeficiency [8]. This suggests that neutrophils play an important role in preventing recurrence of tumors following application of ingenol mebutate. Whether this will translate to a lower rate of recurrence of actinic keratoses in human patients treated with ingenol mebutate remains to be seen.

\section{Clinical Data}

Topical ingenol mebutate gel has been the subject of several recent clinical trials as a potential therapy for actinic keratoses. A review of the literature, including phase II and III clinical trials, suggests that ingenol mebutate is an efficacious and safe treatment for actinic keratosis.

\section{Efficacy}

\section{Phase II Data}

In a multicenter, randomized, double-blinded, vehiclecontrolled, phase IIa study, Siller et al. evaluated the safety and efficacy of ingenol mebutate gel at varying concentrations [12 $]$. A total of 58 Caucasian patients, ranging in age from 44 to 86 years, with at least five clinically diagnosed actinic keratoses on the arms, shoulders, chest, face, or scalp were included. The gel (at concentrations of $0.0025 \%$, $0.01 \%, 0.05 \%$, or placebo) was applied directly to five preselected actinic keratoses on day 1 and day 2 (arm A) or day 1 and day 8 (arm B).

Efficacy, determined by clinical improvement, was evaluated on day 85 . There were no statistically significant differences in the efficacy data from arm A and arm B; thus, the data were combined. Response did vary depending on the concentration of ingenol mebutate gel. Actinic keratoses treated with $0.05 \%$ gel had the highest rates of clinical improvement (53/75 lesions completely cleared, $71 \%)$. A comparison between the vehicle gel placebo group and the treatment groups resulted in a statistically significant $(P<$ $0.0001)$ difference in rates of clinical clearance [12•].

Following early phase I and phase 2a studies supporting the favorable safety profile and appropriate dosing regimen of ingenol mebutate, Anderson et al. conducted a phase $2 b$ study across 22 U.S. centers to further evaluate this promising drug [13]. A total of 222 Caucasian patients, age 4385 years, with 4 to 8 clinically typical actinic keratoses in a $25 \mathrm{~cm}^{2}$ contiguous area on the arm, shoulder, chest, back, or 
scalp were enrolled in a double-blind, double-dummy, vehicle-controlled trial. Patients were randomized to treatment with ingenol mebutate $0.025 \%$ daily for 3 days, ingenol mebutate $0.05 \%$ daily for 3 days, ingenol mebutate $0.05 \%$ for 2 days, or treatment with vehicle gel.

Partial clearance rates (the proportion of patients at day 57 with at least a $75 \%$ reduction in the number of clinically identifiable actinic keratoses) and complete clearance rates (the proportion of patients at day 57 with no clinically visible actinic keratoses in the treatment field) were the primary and secondary end points, respectively. All three treatment arms showed higher clearance rates than the vehicle arm, with a clear dose-response relationship. Partial clearance was seen in $56 \%$ of patients treated with $0.025 \%$ for 3 days, $61.8 \%$ of patients treated with $0.05 \%$ for 2 days, $75.4 \%$ of patients treated with $0.05 \%$ for 3 days, and $21.7 \%$ of the vehicle treatment group. Complete clearance was noted in $40 \%$ of patients treated with $0.025 \%$ for 3 days, $43.6 \%$ of patients treated with $0.05 \%$ for 2 days, $54.4 \%$ of patients treated with $0.05 \%$ for 3 days, and $11.7 \%$ of the vehicle treatment group. Patients treated with ingenol mebutate had a 75-100 \% median percentage reduction in baseline actinic keratosis lesions compared with $0 \%$ for vehicle gel $(P<0.0001$ versus vehicle) [13].

\section{Phase III Data}

Based on encouraging data from phase II studies evaluating the safety and efficacy of ingenol mebutate gel $[12 \bullet, 13]$, phase III trials were initiated in 2008. Lebwohl et al. reported a phase III trial that included four multicenter, randomized, parallel-group, double-blind, vehiclecontrolled studies evaluating both the efficacy and safety of ingenol mebutate gel [3・•]. Caucasian (mostly Fitzpatrick skin types I and II) patients age 18 years and older (mean age 61.5 years), with four to eight discrete actinic keratoses were randomly assigned to self apply ingenol mebutate gel or placebo (vehicle gel) to a $25 \mathrm{~cm}^{2}$ contiguous field. Two of the four trials evaluated patients with actinic keratoses involving the face and scalp with application of ingenol mebutate gel $0.015 \%$ for 3 consecutive days $(n=547)$. The remaining two trials evaluated patients with actinic keratoses on the trunk and extremities with application of ingenol mebutate gel $0.05 \%$ for 2 consecutive days $(n=458)$.

Efficacy was based on the evaluation of patients at baseline and on day 57. Complete clearance of all clinically visible actinic keratoses within the area of treatment was the primary end point. A reduction of at least $75 \%$ of the amount of clinically visible actinic keratoses (partial clearance) on day 57 was defined as the secondary end point.

Complete and partial clearance of actinic keratoses of the face and scalp were significantly higher when ingenol mebutate gel was compared to vehicle gel (placebo): $42 \%$ versus $3.7 \%$ for complete clearance $(P<0.001)$ and $63.9 \%$ versus $7.4 \%$ for partial clearance $(P<0.001)$, respectively $[3 \cdot \bullet]$. Pooled data from the two face and scalp trials showed complete clearance rates were higher for the face $(47.3 \%)$ than for the scalp (22.8\%) [14].

In the trunk and extremity trials, complete and partial clearance of actinic keratoses were significantly higher in the ingenol mebutate group versus the vehicle gel group: $34.1 \%$ versus $4.7 \%$ for complete clearance $(P<0.001)$ and $49.1 \%$ versus $6.9 \%$ for partial clearance $(P<0.001)$ respectively $[3 \cdot \bullet]$. Pooled data from the two trunk and extremity trials showed lower complete clearance rates in the ingenol mebutate group for the back of the hand (18.5\%) versus the arm $(34.5 \%)$ and the chest, leg, back, and shoulder $(60 \%)$ [15].

Long-term follow-up was addressed by this phase III trial in an observational arm involving three of the four study populations, which followed those patients with complete clearance at day 57 for an additional 12 months. At a 12month follow-up of 108 patients who used ingenol mebutate on the face and scalp with complete clearance at day 57, $87.2 \%$ of the resolved actinic keratoses remained clear. One or more new actinic keratoses were observed in $53.9 \%$ of these patients. In 38 patients who received ingenol mebutate to the trunk and extremities with complete clearance by day $57,85.1 \%$ of the resolved actinic keratoses remained clear. One or more new actinic keratoses were observed in $50 \%$ of the patients $[3 \bullet \bullet$.

Schmieder et al. reported a multicenter, open-label, phase $3 b$ study evaluating the safety of ingenol mebutate gel [16]. As a secondary objective, the efficacy of ingenol mebutate gel for the treatment of actinic keratoses on the trunk or extremities was evaluated. The study $(\mathrm{N}=102)$ examined the clearance of actinic keratoses with daily self-applied ingenol mebutate gel $0.05 \%$ for 2 consecutive days. Patients were at least 18 years of age, with 4-8 clinically discrete actinic keratoses within a $25-\mathrm{cm}^{2}$ contiguous field involving the trunk or extremities.

Efficacy was based on clearance and the total percentage of reduction in lesions. At day 57, complete clearance was achieved in $39.2 \%$, and partial clearance (defined as $\geq 75 \%$ clearance) was achieved in $54.9 \%$ of overall patients. The median number of actinic keratoses within the treatment area from baseline to day 57 was reduced by $75 \%$ [16]. Lower rates of clearance were seen in actinic keratoses treated on the dorsal hands, a finding which underscores the difficulty in treating actinic keratoses on the dorsal hands.

\section{Nonmelanoma Skin Cancer}

In addition to its efficacy as a treatment option for actinic keratoses, ingenol mebutate has been shown to be effective 
for the topical treatment of squamous cell carcinoma in situ and squamous cell carcinoma (SCC), both often considered on a continuum with actinic keratoses.

Ramsay et al. conducted a phase I/II clinical study to determine the effectiveness of Euphorbia peplus sap for the topical treatment of squamous cell carcinomas and intraepidermal carcinomas (IEC) (basal cell carcinomas also were included in the study but will not be discussed here) [4]. Twenty patients, at least age 18 years, with biopsyproven IEC $(\mathrm{N}=16)$ or SCC $(\mathrm{N}=4)$ were enrolled in the study. All patients had either failed previous treatments and refused surgery or were considered poor surgical candidates. The IEC/SCC lesions were treated once daily for 3 consecutive days with 100-300 $\mu \mathrm{L}$ of Euphorbia peplus sap. There was a complete response at last follow-up (mean 15 months, range 2-31 months) in IEC and SCC of $75 \%$ and $50 \%$, respectively.

\section{Safety}

In the phase III trial by Lebwohl et al., ingenol mebutate was well tolerated overall. Safety was evaluated the day following the last topical application (day 4 for scalp and face and day 3 for trunk and extremities) and days 8, 15, 29, and 57 [3०0]. As expected, application site adverse reactions were the most commonly reported conditions in all four trials. The trial utilized the local-skin-response scale that graded the following six parameters on a 0 (no reaction) to 4 (severe reaction) scale: erythema, flaking or scaling, crusting, swelling, vesiculation or pustulation, and erosion or ulceration. A composite score was then derived (maximal score was 24). For the face and scalp, the maximal composite score was $9.1 \pm 4.1$ versus $1.8 \pm 1.6$ for the ingenol and placebo groups, respectively. The composite scores for the trunk and extremity groups were $6.8 \pm 3.5$ versus $1.6 \pm 1.5$, respectively. Local skin reactions in patients treated with ingenol mebutate on the face and scalp versus placebo were erythema $(69.7 \%$ vs. $2.2 \%)$, pain $(13.9 \%$ vs. $0.4 \%$ ), pruritus ( $8 \%$ vs. $1.1 \%$ ), and irritation $(1.8 \%$ vs. $0 \%$ ), respectively. In this cohort, the composite score peaked at day 4 and declined to near baseline by day 15 . In the patients treated with ingenol mebutate applied to the trunk and extremities versus placebo, pain $(2.2 \%$ vs. $0 \%)$, pruritus $(8.4 \%$ vs. $0 \%)$, and irritation $(3.6 \%$ vs. $0.4 \%)$ were noted, respectively. The composite score peaked at day 3 for $55.1 \%$, day 8 for $32.4 \%$, and day 15 for $8.4 \%$. Local-skin-response scores were near baseline at day 29 in this group. There were no reported serious adverse events related to the medication. Though not quantified, the authors state there was minimal scarring or change in pigmentation in all four studies $[3 \cdot \bullet]$.

\section{Conclusions}

Actinic keratoses are premalignant lesions that are common in light-skinned populations [17]. There are numerous lesionspecific and field therapies, including cryotherapy, topical imiquimod, topical 5-fluorouracil, topical diclofenac, photodynamic therapy, medium and deep chemical peeling, dermabrasion, and ablative laser resurfacing. The therapy chosen depends on patient characteristics, extent of disease, and tolerability. Ingenol mebutate, the active compound in the sap of Euphorbia peplus, is a new topical therapeutic that has shown efficacy in the treatment of actinic keratoses $[3 \cdot \bullet]$.

There is a proposed dual mechanism of action involving rapid lesion necrosis followed by lesion-specific, neutrophilmediated, antibody-dependent cellular cytotoxity [7••]. However, the precise mechanism of action remains incompletely understood and is the source of ongoing investigations.

The efficacy of ingenol mebutate is comparable to other topical treatments. Imiquimod has complete clearance rates ranging from $30.6 \%(2.5 \%$ for 2 weeks on, 2 weeks off, 2 weeks on) [18] to $35.6 \%$ (3.75\% for 2 weeks on, 2 weeks off, 2 weeks on) [18] to $45.1 \%$ (5\% two times a week for 16 weeks) [19]. One study of 5-fluorouracil daily for 4 weeks showed a complete clearance rate of $47.5 \%$ [20]. The primary benefit of ingenol mebutate is its shorter duration of treatment, which is more convenient for patients and presumably results in higher compliance rates. Further research is needed to compare directly the efficacy of ingenol mebutate gel to other currently available treatment regimens for actinic keratoses.

Ingenol mebutate gel was well-tolerated overall. The primary side effects were local skin reactions that were of short duration. The local-skin-response scores returned to near baseline levels in 15 days for those patients treated on the face and scalp and in 29 days for those patients treated on the trunk and extremities [3••].

The high clearance rates along with a favorable safety profile make ingenol mebutate an important addition to the current therapeutic options for actinic keratoses. Limitations of the medication include lower response rates when applied to the dorsal hands and the scalp compared with other areas of the body. Further research is needed to investigate the application of ingenol mebutate gel on larger areas of skin, because the medication is currently approved for application on areas up to only $25 \mathrm{~cm}^{2}$, as well as to compare directly the safety, efficacy, and tolerability of the medication to other treatments for actinic keratoses.

Conflict of Interest Stephanie Jacks declares that she has no conflict of interest.

Kasie Kudrewicz declares that she has no conflict of interest.

David R. Carr declares that he has no conflict of interest. 


\section{References}

Papers of particular interest, published recently, have been highlighted as:

- Of importance

-• Of outstanding importance

1. Bickers DR, Lim HW, Margolis D, et al. The burden of skin diseases: 2004 a joint project of the American Academy of Dermatology Association and the Society for Investigative Dermatology. J Am Acad Dermatol. 2006;55(3):490-500.

2. Fenske NA, Friedman A, Spencer J. Actinic keratoses: past, present, and future. J Drugs Dermatol. 2010;9(5):s45-9.

3. •- Lebwohl M, Swanson N, Anderson LL, et al. Ingenol mebutate gel for actinic keratosis. N Engl J Med. 2012;366(11):1010-9. The largest phase III trial that included 4 multicenter, randomized, parallel-group, double-blind, vehicle-controlled studies. Demonstrated efficacy of ingenol mebutate for actinic keratoses and a favorable safety profile.

4. Ramsay JR, Suhrbier A, Aylward JH, et al. The sap from Euphorbia peplus is effective against human non-melanoma skin cancers. Br J Dermatol. 2011;164(3):633-6.

5. Green AC, Beardmore GL. Home treatment of skin cancer and solar keratoses. Australas J Dermatol. 1988;29(3):127-30.

6. U.S. Food and Drug Administration. Drugs @ FDA. Available at http://www.accessdata.fda.gov/scripts/cder/drugsatfda/. Accessed January 2013.

7. •- Rosen RH, Gupta AK, Tyring SK. Dual mechanism of action of ingenol mebutate gel for topical treatment of actinic keratoses: rapid lesion necrosis followed by lesion-specific immune response. J Am Acad Dermatol. 2012;66:486-93. This paper reviews the current knowledge on ingenol mebutate's mechanism of action. Discussed is the proposed dual mechanism of action: rapid lesion necrosis followed by lesion-specific neutrophil-mediated, antibody-dependent cellular cytotoxicity.

8. Challacombe JM, Suhrbier A, Parsons PG, et al. Neutrophils are a key component of the antitumor efficacy of topical chemotherapy with ingenol-3-angelate. J Immunol. 2006;177:8123-32.

9. Ogbourne SM, Suhrbier A, Jones B, et al. Antitumor activity of 3ingenyl angelate: plasma membrane and mitochondrial disruption and necrotic cell death. Cancer Res. 2004;64:2833-9.

10. Berman B. Discovery and development timeline of a novel treatment for actinic keratosis: PEP005 (ingenol mebutate) gel. Presented at the 2009 South Beach Symposium. Miami, FL; February 12-16, 2009.
11. Ogbourne SM, Hampson P, Lord JM, et al. Proceedings of the First International Conference on PEP005. Anticancer Drugs. 2007; 18:357-62.

12. - Siller G, Gebauer K, Welburn P, et al. PEP005 (ingenol mebutate) gel, a novel agent for the treatment of actinic keratosis: results of a randomized, double-blind, vehicle-controlled, multicentre, phase IIa study. Australas J Dermatol. 2009;50(1):16-22. This multicenter, randomized, double-blinded, vehicle-controlled, phase IIa study, evaluated the safety and efficacy of ingenol mebutate gel at varying concentrations. Ingenol mebutate was shown to be an effective treatment for actinic keratoses, and the efficacy was dependent on the concentration.

13. Anderson L, Schmieder GJ, Werschler WP, et al. Randomized, double-blind, double-dummy, vehicle-controlled study of ingenol mebutate gel $0.025 \%$ and $0.05 \%$ for actinic keratosis. J Am Acad Dermatol. 2009;60(6):934-43.

14. Berman B, Marmur E, Melgaard A. Three-day topical treatment with ingenol mebutate gel $0.015 \%$ for actinic keratoses on the face and scalp: analysis of data pooled from two trials [Poster P5623] Presented at the Annual Meeting of the American Academy of Dermatology. San Diego, CA; March 16-20, 2012.

15. Anderson LL, Schmeider GJ, Xu Z, Melgaard A. Two-day topical treatment with ingenol mebutate gel $0.05 \%$ for actinic keratoses on the trunk and extremities: analysis of data pooled from two trials [Poster P5640]. Presented at the Annual Meeting of the American Academy of Dermatology. San Diego, CA; March 16$20,2012$.

16. Schmieder G, Xu Z, Larsson T, et al. Efficacy of 2-day ingenol mebutate gel, $0.05 \%$, for actinic keratoses on the trunk or extremities [Poster 5796]. Presented at the Summer Meeting of the American Academy of Dermatology. Boston, Massachusetts; August 15-19, 2012.

17. Rossi R, Mori M, Lotti T. Actinic keratosis. Int J Dermatol. 2007;46(9):895-904.

18. Swanson N, Abramovitx W, Berman B, et al. Imiquimod $2.5 \%$ and $3.75 \%$ for the treatment of actinic keratoses: results of two placebo-controlled studies of daily application to the face and balding scalp for two 2-week cycles. J Am Acad Dermatol. 2010;62(4):582-90.

19. Lebwohl M, Dinehart $S$, Whiting D, et al. Imiquimod $5 \%$ cream for the treatment of actinic keratosis: results from two phase III, randomized, double-blind, parallel group, vehicle-controlled trials. J Am Acad of Dermatol. 2004;50(5):714-21.

20. Weiss J, Menter A, Hevia O, et al. Effective treatment of actinic keratosis with $0.5 \%$ fluorouracil cream for 1,2 , or 4 weeks. Cutis. 2002;70(2):22-9. 